\title{
Role of Spin Momentum Current in Magnetic Non-Local Damping of Ultrathin Film Structures
}

\author{
G. Woltersdorf, R. Urban, and B. Heinrich \\ Simon Fraser University, 8888 University Dr., Burnaby, BC, V5A 1S6, Canada
}

\begin{abstract}
Non-local damping was investigated by Ferromagnetic Resonance (FMR) using ultrathin magnetic single and double layer structures prepared by Molecular Beam Epitaxy (MBE). The double layer structures show magnetic damping which is caused by spin transport across a normal metal spacer $(\mathrm{N})$. In double layer structures a thin Fe layer, F1, was separated from a thick Fe layer, F2, by a $\mathrm{Au}(001)$ spacer. The interface magnetic anisotropies separated the FMR fields of F1 and F2 by a big margin allowing one to investigate FMR in F1 while F2 had a negligible angle of precession, and vice versa. The Fe films in magnetic double layers acquire non-local interface Gilbert damping. It will be shown that the precessing magnetic moments act as spin pumps and spin sinks. This concept was tested by investigating the FMR linewidth around an accidental crossover of the resonance fields for the layers F1 and F2. There is another possible mechanism for non-local damping which is based on a "breathing Fermi surface" of the spacer. The temperature dependence of the non-local damping indicates that this mechanism is weak in Au spacers. Surprisingly the Au spacer acts as an additional impedance for the spin pump mechanism. Finally, it will be shown that electron-electron correlations in a Pd spacer can lead to a significant enhancement of the non-local damping.
\end{abstract}




\section{INTRODUCTION}

The small lateral dimensions of spintronics devices and high density memory bits require the use of magnetic metallic ultrathin film structures where the magnetic moments across the film thickness are locked together by the intra layer exchange coupling. Since spintronics and high density magnetic recording employ fast magnetization reversal processes it is important to understand the spin dynamics and magnetic relaxation processes of multilayers in the nano-second time regime. The spin dynamics is described by the Landau Lifshitz Gilbert (L.L.G.) equation of motion

$$
\frac{1}{\gamma} \frac{\partial \mathbf{M}}{\partial t}=-\left[\mathbf{M} \times \mathbf{H}_{e f f}\right]+\frac{G}{\gamma^{2} M_{s}^{2}}\left[\mathbf{M} \times \frac{\partial \mathbf{M}}{\partial t}\right],
$$

where $\gamma$ is the absolute value of the electron gyromagnetic ratio, $M_{s}$ is the saturation magnetization and $G$ is the Gilbert damping parameter. The effective field $\mathbf{H}_{\text {eff }}$ is given by the derivatives of the Gibbs energy, $U$, with respect to the components $\left(M_{x}, M_{y}, M_{z}\right)$ of the magnetization vector $\mathbf{M}(t)$ [1]. The second term in eq. 1 represents the well known Gilbert damping torque.

\section{NON-LOCAL DAMPING: EXPERIMENT}

The role of non-local damping was investigated in high quality crystalline $\mathrm{Au} / \mathrm{Fe} / \mathrm{Au} / \mathrm{Fe}(001)$ structures grown on $\mathrm{GaAs}(001)$ substrates, see details in [2-5]. In-plane Ferromagnetic Resonance (FMR) experiments were carried out using 10, 24, 36 and $72 \mathrm{GHz}$ systems $[4,6]$.

Single Fe ultrathin films with thicknesses of 8, 11, 16, 21, and 31 monolayers (ML) were grown directly on $\mathrm{GaAs}(001)$ and covered by a $20 \mathrm{ML}$ thick $\mathrm{Au}(001)$ cap layer for protection in ambient conditions. FMR measurements were used to determine the in-plane four-fold and uniaxial magnetic anisotropies, $K_{1}$ and $K_{u}$, and the effective demagnetizing field perpendicular to the film surface, $4 \pi M_{e f f}$, as a function of the film thickness $d[6]$. The magnetic anisotropies were well described by the bulk and interface magnetic properties, respectively [2]. The thin Fe films which were studied in the single layer structures were regrown as a part of magnetic double layer structures. The thin Fe film (F1) was separated from the second thick Fe layer (F2) by a $\mathrm{Au}(001)$ spacer $(\mathrm{N})$ of a variable thickness between 8 to $100 \mathrm{ML}$. The magnetic double layers were covered by a $20 \mathrm{ML} \mathrm{Au}(001)$ capping layer. 
The thickness of the Au spacer layer was always smaller than the electron mean free path (38 nm) [7], and hence allowed ballistic spin transport between the magnetic layers.

The interface magnetic anisotropies separated the FMR fields of F1 and F2 by a big margin ( 1 kOe), see Fig.1 allowing one to carry out FMR measurements in F1 with F2 possessing a small angle of precession compared to that in F1, and vice versa. The thin Fe film in the single and double layer structures had the same FMR field showing that the static interlayer exchange coupling in the double layer [2] through the Au spacer was negligible. The absence of static interlayer coupling is due to short atomic terraces [1].

The results are as follows: (a) The FMR linewidth in the thin films F1 always increased in the presence of F2, and vice versa; (b) The additional FMR linewidth, $\Delta H_{a d d}$, followed an inverse dependence on the thin film thickness $d_{1}$, see [2]; and (c) the additional FMR linewidth $\Delta H_{a d d}$ in both the parallel ( $\mathrm{H}$ in-plane) and perpendicular ( $\mathrm{H}$ perpendicular to the plane) FMR configuration was linearly dependent on the microwave frequency with no zero frequency offset [5]. The additional Gilbert damping for the $16 \mathrm{ML}$ thick Fe film was found to be only weakly dependent on the crystallographic direction, with the average value $G_{a d d}=1.2 \times 10^{8} \mathrm{~s}^{-1}$. This is comparable to the intrinsic Gilbert damping in the single Fe film, $G_{\text {int }}=1.4 \times 10^{8} \mathrm{~s}^{-1}$.

\section{THEORY OF NON-LOCAL DAMPING}

Tserkovnyak et al. [8] showed that the interface damping can be generated by spin current from a ferromagnet $(\mathrm{F})$ into the adjacent normal metal reservoirs (NM). The spin current is generated by a precessing magnetic moment. A precessing magnetization at the $\mathrm{F} / \mathrm{NM}$ interface acts as a "peristaltic spin -pump". The direction of the spin current is perpendicular to the F/NM interface and points away from the interface towards NM. The spin momentum which is carried away by the spin current is

$$
\mathbf{j}_{\text {spin }}=\frac{\hbar}{4 \pi} A_{r} \mathbf{m} \times \frac{d \mathbf{m}}{d t},
$$

where $\mathbf{m}$ is the a unit vector in direction of $\mathbf{M}$. The spin current can cause magnetic damping. $A_{r}$ is the interface scattering parameter, and for $\mathrm{F}$ films thicker than spin coherence length, $\pi /\left(k_{F}^{\uparrow}-k_{F}^{\downarrow}\right)$, given by

$$
A_{r}=\frac{1}{2} \sum_{m, n}\left|r_{m n}^{\uparrow}-r_{m n}^{\downarrow}\right|^{2}
$$



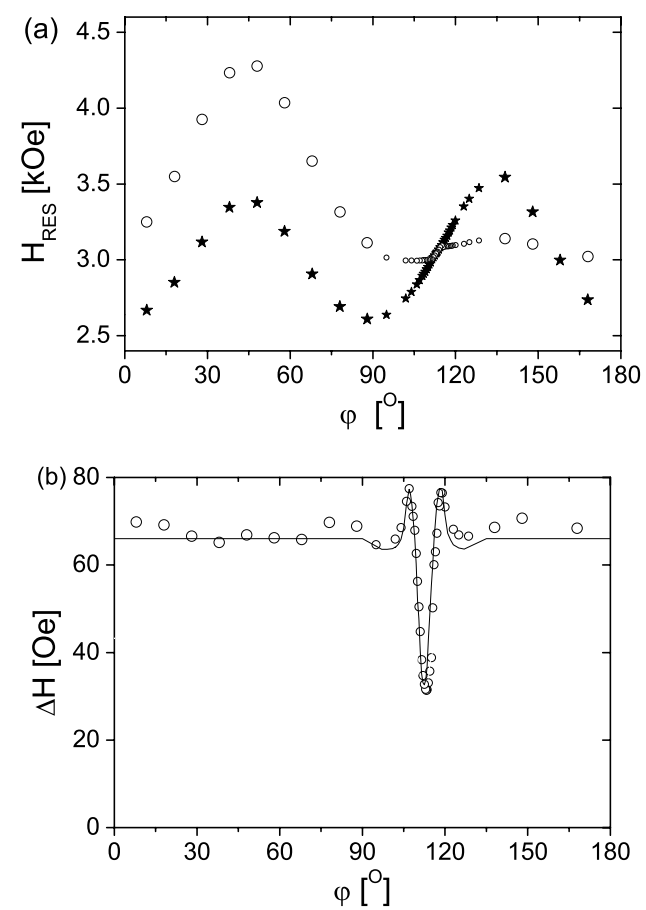

FIG. 1: (a) The FMR fields at $24 \mathrm{GHz}$ in the layer F1(16Fe, shown by (o)) and layer F2(40Fe, $(\star)$ ) in $20 \mathrm{Au} / 40 \mathrm{Fe} / 16 \mathrm{Au} / 16 \mathrm{Fe} / \mathrm{GaAs}(001)$ (integers represent the number of atomic layers) as a function of the angle $\varphi$ between the applied field and the in-plane [100] crystallographic direction. A large in-plane uniaxial anisotropy field in F1 leads to an accidental crossover at $\varphi=115$ and 150 Deg. Notice that the FMR fields get locked together by the spin pumping effect at the accidental crossover. Away from the crossover the resonance fields are separated by as much as ten FMR linewidths.

(b) The FMR linewidths corresponding to F1 in Fig.1(a). The measured and calculated FMR signals were analyzed using two Lorenzian lineshapes. The Lorenzian peaks were characterized by their amplitudes, resonance fields and linewidths. The solid lines were obtained from calculations using eqs 7 . Note that the FMR linewidth for F1 first increases before it reaches its minimum value corresponding to that of a single layer structure.

where $r_{m n}^{\uparrow \downarrow}$ are the electron reflection matrix elements at the NM/F interface for the spin up and down electrons. $k_{F}^{\uparrow, \downarrow}$ are the Fermi $k$ vectors for majority and minority electrons, respectively. The sum in $A_{r}$ is close to the number of the transverse channels in NM [9]. 

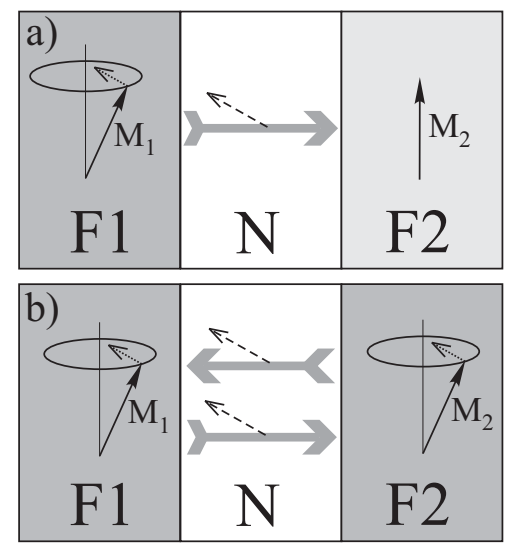

FIG. 2: A cartoon representing the dynamic coupling between two magnetic layers which are separated by a non-magnetic spacer N. (a) represents two magnetic layers with different FMR fields. F1 is at resonance, and F2 is nearly stationary. A large grey arrow in the normal spacer describes the direction of the spin current. The dashed lines represent the instantaneous direction of the spin momentum. For small angle of precession they are nearly parallel to the transverse rf magnetization component shown in short solid arrows. F1 acts as a spin pump, F2 acts as a spin sink. (b) represents a situation when $\mathrm{F} 1$ and F2 resonate at the same field. Both layers act as spin pumps and spin sinks. In this case the net spin momentum transfer across each interface is zero. No additional damping is present.

The sum is given by

$$
\frac{A_{r}}{S}=\frac{k_{F}^{2}}{4 \pi}=0.85 n^{2 / 3}
$$

where $S$ is the area of the interface, $k_{F}$ is the Fermi wavevector and $n$ is the density of electrons per spin in NM [9]. Brataas et al. [9, 10] showed that $A_{r}$ can be evaluated from the interface mixing conductance $G_{\uparrow \downarrow}[11] . A_{r}=\frac{h}{e^{2}} G_{\uparrow \downarrow}=S g_{\uparrow \downarrow}$, where $g_{\uparrow \downarrow}$ represents "dimensionless interface mixing conductivity".

The generated spin current propagates though the normal metal spacer N, and is deposited at the N/F2 interface. It was shown [10, 12] that the transverse component of the spin current in $\mathrm{N}$ is entirely absorbed at the N/F2 interface, see Fig.2a. For small precessional angles the spin current is almost entirely transverse. This means that the N/F2 interface acts as an ideal spin sink, and provides an effective spin brake for the precessing magnetic moment in $\mathrm{F} 1[4,5,13]$. The spin momentum $\mathbf{j}_{\text {spin }}$ which is carried away by the spin current has the form of Gilbert damping. The resulting Gilbert damping is given by 
the conservation of the total spin momentum

$$
\mathbf{j}_{\text {spin }}-\frac{1}{\gamma} \frac{\partial \mathbf{M}_{t o t}}{\partial t}=0
$$

where $\mathbf{M}_{t o t}$ is the total magnetic moment in F1. After simple algebraical steps one obtains an expression for the dimensionless spin pump contribution to the damping, $\alpha_{s p}$,

$$
\alpha_{s p}=\frac{G_{s p}}{\gamma M_{s}}=g \mu_{B} \frac{g_{\uparrow \downarrow}}{4 \pi M_{s}} \frac{1}{d_{1}},
$$

where $d_{1}$ is the thickness of $\mathrm{F} 1, g_{\uparrow \downarrow}$ is the dimensionless mixing conductivity, and $G_{s p}$ is the spin pump Gilbert parameter. $g$ is the electron g-factor. The inverse dependence of $\alpha_{s p}$ on the film thickness clearly testifies to its interfacial origin. The layers F1 and F2 act as mutual spin pumps and spin sinks. For small precessional angles the eq. of motion for F1 can be written as [4]

$$
\begin{gathered}
\frac{1}{\gamma} \frac{\partial \mathbf{M}_{1}}{\partial t}=-\left[\mathbf{M}_{1} \times \mathbf{H}_{e f f, 1}\right]+\frac{G_{1}}{\gamma^{2} M_{s}^{2}}\left[\mathbf{M}_{1} \times \frac{\partial \mathbf{M}_{1}}{\partial t}\right] \\
+\frac{\hbar}{4 \pi d_{1}} g_{\uparrow \downarrow, 1} \mathbf{m}_{1} \times \frac{\partial \mathbf{m}_{1}}{\partial t}-\frac{\hbar}{4 \pi d_{1}} g_{\uparrow \downarrow, 2} \mathbf{m}_{2} \times \frac{\partial \mathbf{m}_{2}}{\partial t},
\end{gathered}
$$

where $\mathbf{M}_{1}$ is the magnetization vector of $\mathrm{F} 1, \mathbf{m}_{1,2}$ are the unit vectors along $\mathbf{M}_{1,2}$, and $d_{1}$ is the thicknesses of F1. The exchange of spin currents is a symmetric concept and the eq. of motion for the layer F2 is obtained by interchanging the indices $1 \rightleftarrows 2$. The third and fourth terms in eq. 7 represent the spin pump and spin sink of F1. The fourth term is generated by the spin pump from F2. For clarity it is worthwhile to point out that the signs $(+)$ and $(-)$ in the third and fourth terms in eq. 7 represent the spin current directions $(\mathrm{F} 1 \rightarrow \mathrm{F} 2)$ and $(\mathrm{F} 2 \rightarrow \mathrm{F} 1)$, respectively.

The spin pump model is a rather exotic theory to those who are working in magnetism. One would expect that there is a direct connection to a more common concept which is applicable to magnetic multilayers. The obvious choice is interlayer exchange coupling. In the past interlayer exchange interaction was treated only in the static limit [14]. Recently it was shown that its dynamic part can create magnetic damping [4, 13, 15]. One can show that the spin pumping theory is equivalent to the dynamic response of the interlayer exchange coupling. Since the dynamic coupling is derived from the rf susceptibility one can account for electron-electron correlation effects in the normal metal spacer [13]. It has been shown [15] that the Gilbert damping is enhanced by the square of the Stoner factor 
$S_{E}=\left[1-U N\left(E_{F}\right)\right]^{-1}$,

$$
G_{s-d}^{e n h}=G_{s-d} S_{E}^{2},
$$

where $U$ is the screened interatomic Coulomb interaction and $N\left(E_{F}\right)$ the electron density of states, per atom, at the Fermi level in NM.

It is worthwhile to realize that the $s-d$ exchange relaxation mechanism also applies to bulk ferromagnets, and was evaluated by Heinrich et al. [16, 17]. The Gilbert damping in this case is given by

$$
G_{s-d}^{b u l k}=\frac{\chi_{P}}{\tau_{s f}}
$$

where $\chi_{P}$ is the Pauli susceptibility and $\tau_{s f}$ is the spin flip relaxation time of itinerant electrons in the ferromagnet. It should be noted that $1 / \tau_{s f}$ in metals is proportional to the square of the spin orbit interaction $[16,17]$. Using $\chi_{P}$ from Kriesman and Callen [18] and $\tau_{s f}$ from the spin diffusion length in Current Perpendicular to Plane (CPP) GMR measurements one obtains for the bulk Gilbert damping $G=5 \times 10^{6} \mathrm{~s}^{-1}$ and $1 \times 10^{8} \mathrm{~s}^{-1}$ for Co and permalloy (Py), respectively, see the details in [17]. This contribution is small in Co but it explains the intrinsic damping in Py. Fe is expected to behave like Co.

One expects that there has to be an additional mechanism which depends explicitly on $\tau_{s f}$. Its origin can be understood in the following way. The mechanism of the interlayer exchange coupling lies in the itinerant nature of the electron carriers [13]. The energy of electrons depends on the instantaneous orientation of the magnetic moments, and consequently the occupation number $n_{\mathrm{k}, \sigma}$ of electronic states having energy $\varepsilon_{\mathrm{k}, \sigma}$ changes during precession of the magnetization, and this results a "breathing Fermi surface", this concept was also used in bulk materials [19, 20]. However, this redistribution cannot be achieved instantaneously. The time lag between the instantaneous exchange field and the induced moment in the spacer is described by the transverse spin relaxation time, $\tau_{s f}$, which is proportional to the momentum relaxation time entering conductivity [21]. In the limit of slow precessional motion the effective damping field can be evaluated $[4,13]$,

$$
\mathbf{H}_{\text {damp }}^{\text {int }}=\tau_{s f} \sum_{\mathrm{k}, \sigma} \delta\left(\varepsilon_{\mathrm{k}, \sigma}\left[\mathbf{M}_{\mathbf{1}}\right]-\varepsilon_{F}\right)\left(\frac{\partial \varepsilon_{\mathrm{k}, \sigma}\left[\mathbf{M}_{\mathbf{1}}\right]}{\partial \mathbf{M}_{\mathbf{1}}}\right)^{2} \frac{1}{d} \frac{\partial \mathbf{M}_{\mathbf{1}}}{\partial t},
$$

where the sum is carried out per unit area. In this case the damping field is proportional to the spin relaxation time $\tau_{s f}$. This mechanism is explicitly dependent on the conductivity and it represents a different contribution to the non-local damping compared to the spin pumping mechanism which is independent on $\tau_{s f}$. 


\section{DISCUSSION OF THE RESULTS}

The spin pumping and breathing Fermi surface theories predict Gilbert damping, which results in a strictly linear dependence of $\Delta H_{a d d}$ on the microwave frequency. This is indeed experimentally observed over a wide range of microwave frequencies [3, 4].

The validity of the spin-pumping theory can be tested by comparing calculations using eqs 7 with the experimental results. Fig.2 shows two extreme situations. In Fig. 2(a) the FMR fields in F1 and F2 are separated by a big margin. In Fig.2(b) the FMR fields are same. In (a) one expects the full contribution from the non-local damping. $\Delta H_{a d d}$ for F1 and F2 should scale with their respective $1 / d$ terms. In (b) the situation is symmetric; the net spin momentum current trough both interfaces is zero, and the additional damping is absent. This behavior is well demonstrated in Fig.1b. The good agreement between theory and experiment clearly shows the validity of the spin pumping and spin sink concept which is described by eqs 7 . Even in the absence of static interlayer exchange coupling the magnetic layers are coupled by the dynamic part of the interlayer exchange. The spin sink effect at the N/F interface starts to be inefficient only when the thickness of the normal metal spacer $\mathrm{N}$ becomes comparable to the spin diffusion length [22]. Since the resistivity ratio in our structures is a factor of two, see Fig.4 the spin diffusion length in Au is at least of the order of $100 \mathrm{~nm}$. The static interlayer exchange coupling vanishes in our samples due to interface roughness on a length scale of a mere $10 \mathrm{ML}(2 \mathrm{~nm})$. One should point out that when the $\mathrm{N}$ metal spacer thickness starts to be comparable to the spin diffusion length then the $\mathrm{N}$ spacer absorbs a part of the spin current [23, 24].

The quantitative comparison with predictions of the spin pumping theory is very favorable. First principles electron band calculations [11] resulted in $g_{\uparrow \downarrow} \approx 1.11 \times 10^{15} \mathrm{~cm}^{-2}$ for a clean $\mathrm{Cu} / \mathrm{Co}(111)$ interface. By scaling this value to Au using eq.4 one obtains $G_{s p}=1.37 \times 10^{8} \mathrm{~s}^{-1}$ which is only $14 \%$ higher than the value measured at room temperature. This is surprising agreement considering the fact that calculations of the intrinsic damping in bulk metals have been carried out over the last three decades, and yet they have not been able to produce a comparable agreement with experiments [17].

The breathing Fermi surface contribution to the Gilbert damping is proportional to the electron relaxation time $\tau_{s f}$ of the $\mathrm{N}$ metal spacer, see eq.10. Since this contribution is using the concept of interlayer exchange coupling one can expect some degree of oscillatory 


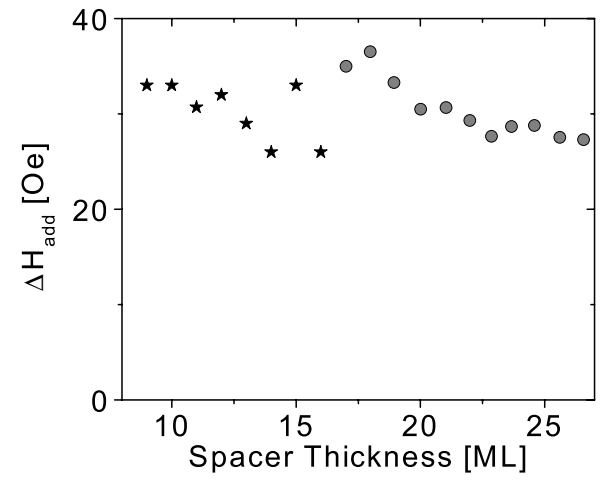

FIG. 3: The additional FMR linewidth, $\Delta H_{a d d}$, as function of the Au spacer thickness in $20 \mathrm{Au} / 8-$ $27 \mathrm{Au} / 16 \mathrm{Fe} / \mathrm{GaAs}(001)$. The samples with the Au spacer thickness between 8 to $16 \mathrm{ML}$ were grown separatelly. The samples with the spacer thickness between 17 and $27 \mathrm{ML}$ were grown using a wedged $\mathrm{Au}(001)$ layer. One can see some degree of variation in $\Delta H_{a d d}$ with a period of $\sim 3 \mathrm{ML}$. These oscillations are marginal.

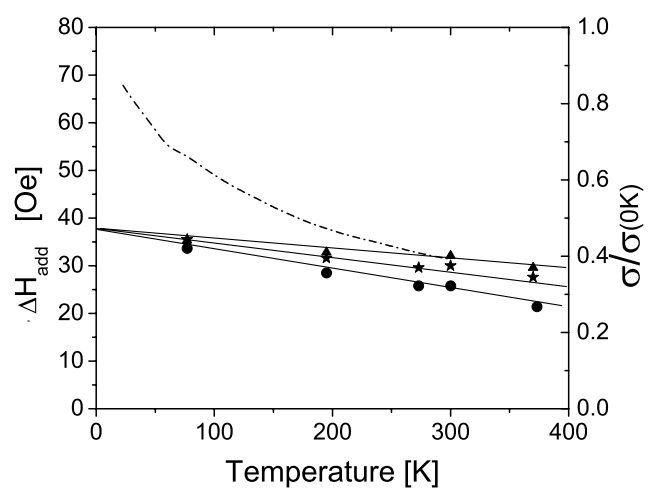

FIG. 4: The temperature dependence of the additional FMR linewidth, $\Delta H_{\text {add }}$, for 3 different samples: $(\boldsymbol{\Delta}) 20 \mathrm{Au} / 40 \mathrm{Fe} / 14 \mathrm{Au} / 16 \mathrm{Fe} / \mathrm{GaAs}(001),(\star) 20 \mathrm{Au} / 40 \mathrm{Fe} / 40 \mathrm{Au} / 16 \mathrm{Fe} / \mathrm{GaAs}(001)$, and $(\bullet)$ $20 \mathrm{Au} / 40 \mathrm{Fe} / 100 \mathrm{Au} / 16 \mathrm{Fe} / \mathrm{GaAs}(001)$. The temperature dependence of the sheet conductivity, $\sigma$, for the $(\bullet)$-sample is shown in the dashed line. Note that the temperature dependence of $\Delta H_{a d d}$ is linear and weak for all samples. One can fit the temperature dependence by eq. 11 .

behavior with a changing spacer thickness. The measurements shown in Fig. 3 indicate that this behavior is only weakly present. A better test of the breathing Fermi surface contribution can be carried out by measuring the temperature dependence of the non-local damping. One expects proportionality with the sheet conductance $\left(\tau_{s f} \sim \tau_{\text {orb }} \sim \sigma\right)$ of the 


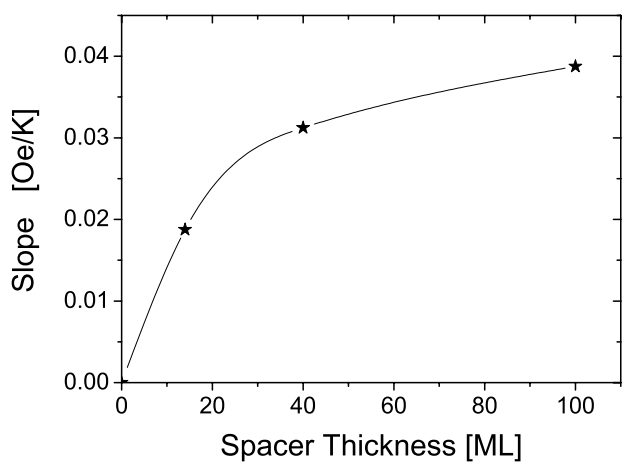

FIG. 5: The slope, $\left(\partial \Delta H_{a d d} / \partial T\right)$, of the additional FMR linewidth, as function of the spacer thickness. The slope was obtained for the samples shown in Fig.4. The solid line is a spline fit to guide the reader's eye.

$\mathrm{N}$ spacer. The temperature dependence of the sheet conductance and the additional FMR linewidth is shown in Fig. 4. The temperature dependence od $\Delta H_{\text {add }}$ is linear and obviously does not scale with the conductivity. This clearly indicates that the strength of the breathing Fermi surface contribution is unimportant in the $\mathrm{Fe} / \mathrm{Au} / \mathrm{Fe}(001)$ samples. Note, that the slope of $\Delta H_{a d d}$ as a function of temperature increases with an increasing spacer thickness. One can fit the temperature dependence by the linear function

$$
\Delta H_{a d d}=\text { Slope } \cdot \mathrm{T}+\Delta H_{a d d}(\mathrm{~T}=0 \mathrm{~K})
$$

Note, that $\Delta H_{a d d}(\mathrm{~T}=0 \mathrm{~K})$ is equal for all measured $\mathrm{Au}$ spacers. Fig. 5 shows the fitted slope as function of the spacer thickness. One can see that the slope saturates for higher spacer thicknesses. This implies that a part of the Au spacers at finite temperatures decreases effectiveness of the spin pump mechanism and acts as an additional resistance in series with the interface mixing resistance $1 / g_{\uparrow \downarrow}$. This decreases the spin current reaching the N/F2 interface. The additional resistance in Au decreases with decreasing temperature and becomes zero for $T=0 \mathrm{~K}$. Since the spin diffusion length in $\mathrm{Au}$ is much bigger than the $\mathrm{Au}$ spacer thickness no additional resistance in $\mathrm{Au}$ is expected. The linear temperature dependence of $\Delta H_{a d d}(\mathrm{~T})$ can be indicative that the Au resistance follows the temperature dependence of inverse susceptibility $(1 / \chi \sim \mathrm{T})$ of "loose spins" [1, 25]. The impurity Fe atoms (loose spins) can be interdiffused up to certain depth (see saturation of this effect with increasing $\mathrm{Au}$ spacer thickness in Fig. 3) around the F1/N interface by surfactant Arsenic during the deposition of $\mathrm{Au}$ on $\mathrm{F} 1$ [7]. Using this picture one can argue that the 
temperature dependence of $\Delta H_{\text {add }}(\mathrm{T})$ in Fig. 3 could be a consequence of "loose spins" which contribute to the impedance of the spin pumping mechanism. It is worthwhile to note that the measured value of $G_{s p}=1.4 \times 10^{8} \mathrm{~s}^{-1}$ at $\mathrm{T}=0 \mathrm{~K}$ is very close to that estimated from the spin pumping theory, $G_{s p}=1.37 \times 10^{8} \mathrm{~s}^{-1}$.
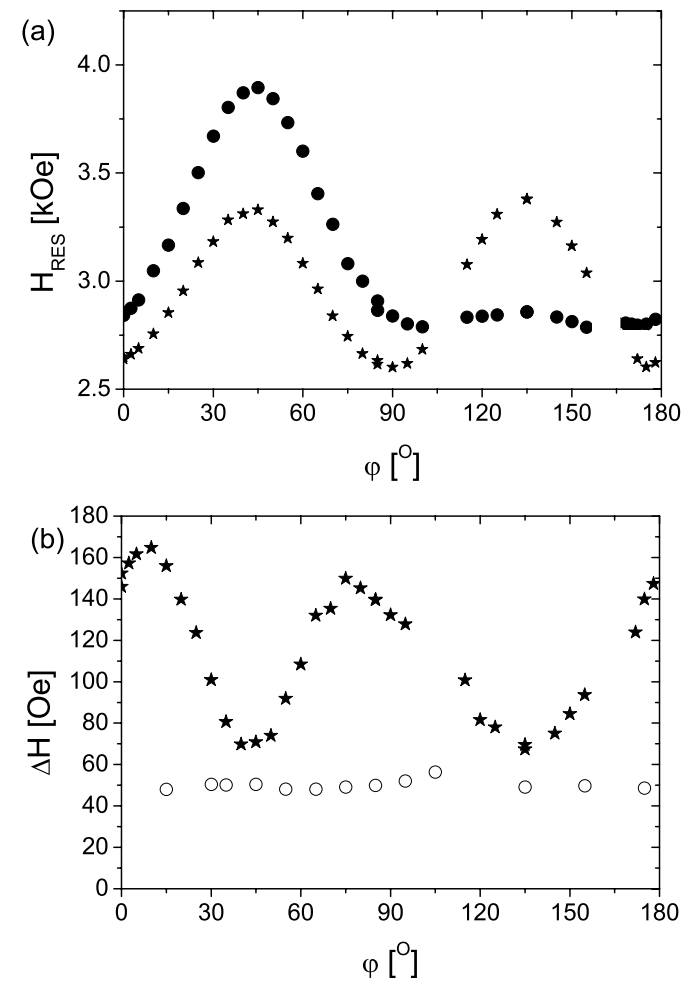

FIG. 6: (a) The FMR fields of the two Fe layers in the GaAs/16Fe/9Pd/40Au/40Fe/20Au(001) sample. $(\bullet)$ and $(\star)$ correspond to $16 \mathrm{Fe}$ and $40 \mathrm{Fe}$, respectively. The measurements where carried out at $24 \mathrm{GHz}$. (b) shows the FMR linewidth for $16 \mathrm{Fe}$ in single [GaAs/16Fe/9Pd/20Au(001)] (o), and double layer $[\mathrm{GaAs} / 16 \mathrm{Fe} / 9 \mathrm{Pd} / 40 \mathrm{Au} / 40 \mathrm{Fe} / 20 \mathrm{Au}(001)](\bullet)$ sample. We did not analyze the FMR data around the accidental crossover of resonance fields at 110 and 165 degrees, respectively. Around these points the two FMR peaks resulted in a broad and asymmetric spectrum not allowing one to carry out a reliable analysis.

The dynamic exchange coupling theory [13], includes the Stoner enhancement factor, see eq.8. Our recent results using $20 \mathrm{Au} / 4 \mathrm{Pd} /[\mathrm{Fe} / \mathrm{Pd}]_{5} / 14 \mathrm{Fe} / \mathrm{GaAs}(001)$ single and $20 \mathrm{Au} / 40 \mathrm{Fe} / 40 \mathrm{Au} / 4 \mathrm{Pd} /[\mathrm{Fe} / \mathrm{Pd}]_{5} / 14 \mathrm{Fe} / \mathrm{GaAs}(001)$ double layer samples, see [13], show a strong evidence for the Stoner enhancement factor. This structure incorporates a magnetic $[\mathrm{Fe} / \mathrm{Pd}]_{5}$ superlattice with 5 repetitions of a $[1 \mathrm{Fe} / 1 \mathrm{Pd}]$ unit cell. The $\mathrm{N}$ metal spacer 
is $4 \mathrm{Pd} 40 \mathrm{Au}(001)$. The additional FMR linewidth along the cubic crystallographic axes was enhanced by as much as a factor of four, see [4, 13] and is significantly bigger than that expected from the simple spin pumping mechanism. It turns out that one can get the same enhancement in non-local damping using a simple GaAs/16Fe/9Pd/40Au/40Fe/20Au(001) double layer structure, see Fig. 6 where the Fe atomic layers were removed from the $[\mathrm{Fe} / \mathrm{Pd}]_{5}$ superlattice. In this case the single layer structure is GaAs/16Fe/9Pd/20Au(001) where no enhancement of the damping was observed, see Fig. 6. The metallic Pd is known to exhibit a strong Stoner enhancement in the dc susceptibility [26]. The Fermi surface of Pd is not spherical and can lead to a significant angular dependence of the Stoner enhancement factor which satisfies the (001) symmetry of our samples, see Fig. 6. These results clearly show that electron correlation effects in normal metal spacers have to be considered.

\section{CONCLUSIONS}

We have shown that non-local damping by the transfer of spin momentum can be realized in magnetic multilayer films. This effect is significant in ultrathin films. Theoretical models were presented for the non-local damping. It has been demonstrated that the non-local interface Gilbert damping in magnetic multilayers is well described by the concept of spin pumps and spin sinks. It has been shown that this effect is directly related to the dynamics of the interlayer exchange coupling. By proper engineering of multilayer structures one can create magnetic damping which significantly surpasses that in the bulk materials.

The Authors thank Y. Tserkovnyak, A. Brataas, G.E.W. Bauer, J. F. Cochran, D. Riemann, E. Rozenberg, and K. Myrtle for their assistance and valuable discussions during the course of this work. Financial support from the Natural Sciences and Engineering Research Council of Canada (NSERC) and Canadian Institute for Advanced Research (CIAR) is gratefully acknowledged. G.W. thanks the German Academic Exchange Service (DAAD) for generous financial support.

[1] B. Heinrich and J. F. Cochran, Adv. Phys. 42, 523 (1993).

[2] R. Urban, G. Woltersdorf, and B. Heinrich, Phys. Rev. Lett. 87, 217204 (2001).

[3] B. Heinrich, R. Urban, and G. Woltersdorf, IEEE Trans. Mag. 38, 2496 (2002). 
[4] B. Heinrich, G. Woltersdorf, R. Urban, and E. Simanek, Conference on Mag. Mag. Mat., Tampa, Florida, 2002, J. Appl. Phys. (submitted).

[5] B. Heinrich, R. Urban, and G. Woltersdorf, J. Appl. Phys. 91, 7523 (2002).

[6] B. Heinrich and J. A. C. Bland, Ultrathin Magnetic Structures II. (Spinger-Verlag, 1994), section Radio Frequency Techniques.

[7] A. Enders, T. Monchesky, K. Myrtle, R. Urban, B. Heinrich, J. Kirschner, X.-G. Zhang, and W. Butler, J. Appl. Phys. 89, 7110 (2001).

[8] Y. Tserkovnyak, A. Brataas, and G. Bauer, Phys. Rev. Lett. 88, 117601 (2002).

[9] A. Brataas, Y. Tserkovnyak, G. Bauer, and B. Halperin, Phys. Rev. B 66, id060404 (2002).

[10] A. Brataas, Y. Nazarov, and G. Bauer, Erp. Phys. J. B 22, 99 (2001).

[11] K. Xia, P. Kelly, G. Bauer, A. Brataas, and I. Turek, Phys.Rev. B 65, 220401 (R) (2002).

[12] M. Stiles and A. Zangwill, J.Appl.Phys. 91, 6812 (2002).

[13] B. Heinrich, G. Woltersdorf, R. Urban, and E. Simanek, Moscow International Symposium on Magnetism, Moscow 2002, J. Mag. Mag. Mat. (in press).

[14] J. Slonczewski, J. Mag. Mag. Mater. 126, 374 (1993).

[15] E. Simanek and B. Heinrich, arXiv:cond-mat/0207471 (2002).

[16] B. Heinrich, D. Fraitová, and V. Kamberský, Phys. Stat. Sol. 23, 501 (1967).

[17] B. Heinrich and J. A. C. Bland, Ultrathin Magnetic Structures III (Springer Verlag, 2003), chap. B. Heinrich on Spin Relaxations in Magnetic Metallic Layers and Multilayers.

[18] C. Kriesman and H. Callen, Phys. Rev. 94, 837 (1954).

[19] V. Korenman and R. Prange, Phys. Rev. B 6, 2769 (1972).

[20] V. Kamberský, Can. J. Phys. 48, 2906 (1970).

[21] R. J. Elliot, Phys. Rev. 96, 266 (1954).

[22] R. Silsbee, A. Janossy, and P. Monod, Phys. Rev. B 19, 4382 (1979).

[23] S. Mizukami, Y. Ando, and T. Miyazaki, J. Mag. Mag. Mat. 226, 1640 (2001).

[24] R. Urban, G. Woltersdorf, and B. Heinrich, J.Appl.Phys. (submitted).

[25] J. Slonczewski, J. Appl. Phys. 73, 5957 (1993).

[26] M. Brodsky and A. Freeman, Phys. Rev. Lett. 45, 133 (1980). 